
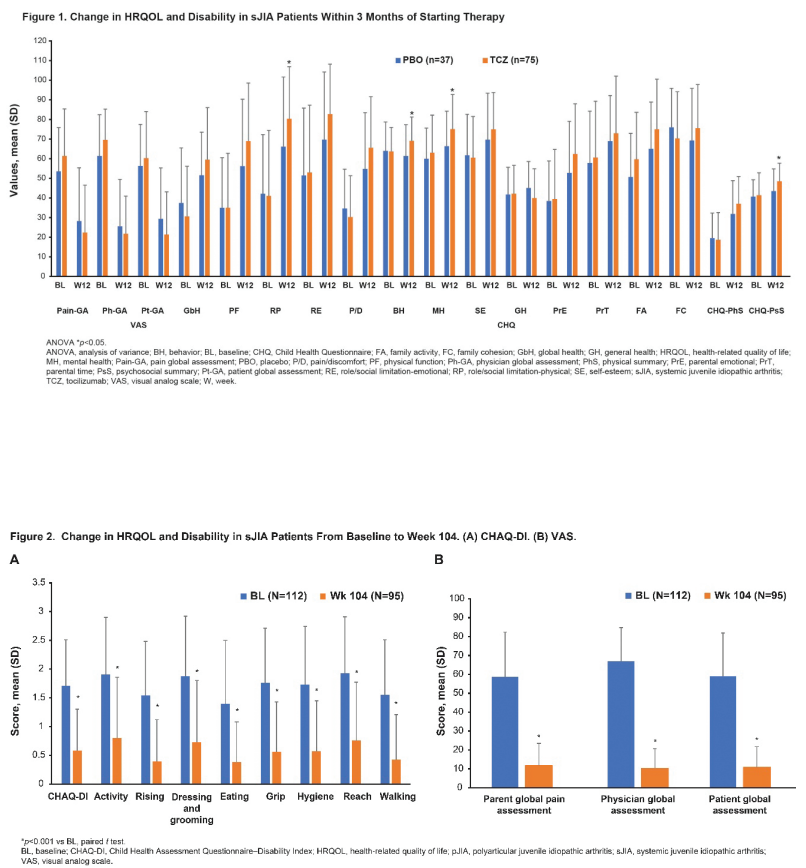

Disclosure of Interests: Nicolino Ruperto Grant/research support from: The Gaslini Hospital, where NR works as full-time public employee, has received contributions (> 10.000 USD each) from the following industries in the last 3 years: BMS, Eli-Lilly, GlaxoSmithKline, F Hoffmann-La Roche, Janssen, Novartis, Pfizer, Sobi. This funding has been reinvested for the research activities of the hospital in a fully independent manner, without any commitment with third parties., Consultant for: Received honoraria for consultancies or speaker bureaus (< 10.000 USD each) from the following pharmaceutical companies in the past 3 years: Ablynx, AbbVie, Astrazeneca-Medimmune, Biogen, Boehringer, Bristol-Myers Squibb, Eli-Lilly, EMD Serono, GlaxoSmithKline, Hoffmann-La Roche, Janssen, Merck, Novartis, Pfizer, R-Pharma, SanofiServier, Sinergie, Sobi and Takeda., Speakers bureau: Received honoraria for consultancies or speaker bureaus ( $<10.000$ USD each) from the following pharmaceutical companies in the past 3 years: Ablynx, AbbVie, Astrazeneca-Medimmune, Biogen, Boehringer, Bristol-Myers Squibb, Eli-Lilly, EMD Serono, GlaxoSmithKline, Hoffmann-La Roche, Janssen, Merck, Novartis, Pfizer, RPharma, SanofiServier, Sinergie, Sobi and Takeda., Chen Chen: None declared, Alberto Martini: None declared, Graciela Espada: None declared, Rik Joos: None declared, Jonathan Akikusa: None declared, Jeffrey Chaitow: None declared, Maria Luz Gámir Gámir: None declared, Yukiko Kimura Consultant for: Novartis, SOBI, Christoph Rietschel: None declared, Daniel Siri: None declared, Elzbieta Smolewska: None declared, Heinrike Schmeling Grant/research support from: F. Hoffmann-La Roche Ltd, Diane Brown: None declared, Fabrizio De Benedetti Grant/research support from: Abbvie, SOBI, Novimmune, Roche, Novartis, Sanofi, Pfizer, Daniel J Lovell Consultant for: Consulting fees and/or honoraria from Astra Zeneca, Wyeth Pharma, Amgen, Abbott, Pfizer, F. Hoffmann-La Roche, Novartis, UBC, Takeda, GSK, Boehringer, and Celgene, Bin Huang: None declared, Hermine Brunner Grant/research support from: Bristol-Myers Squibb, Pfizer, Consultant for: Pfizer, Bristol-Myers Squibb, Janssen, Novartis, Lilly, Roche, GlaxoSmithKline, Sanofi, Speakers bureau: Novartis, Roche

DOI: 10.1136/annrheumdis-2019-eular.3392

\section{FRI0573 \\ COGNITIVE IMPAIRMENT IN CHILDHOOD-ONSET SYSTEMIC LUPUS ERYTHEMATOSUS: EARLY DETECTION WITH MR SPECTROSCOPY AND ITS ASSOCIATION WITH MOG ANTIBODIES}

Huseyin Kilic ${ }^{1}$, Sezgin Sahin ${ }^{2}$, Mekiya Filiz Toprak ${ }^{3}$, Gokce Hale Hatay ${ }^{4}$, Kubra Yilmaz ${ }^{1}$, Amra Adrovic ${ }^{2}$, Kenan Barut ${ }^{2}$, Esin Ozturk Isik ${ }^{4}$, Erdem Tuzun ${ }^{5}$, Osman Kizilkilic ${ }^{6}$, Sema Saltik ${ }^{7}$, Ozgur Kasapcopur ${ }^{2}$. ${ }^{1}$ Istanbul UniversityCerrahpasa, Cerrahpasa Medical School, Pediatric Neurology, Istanbul, Turkey; ${ }^{2}$ Istanbul University-Cerrahpasa, Cerrahpasa Medical School, Pediatric Rheumatology, İstanbul, Turkey, ${ }^{3}$ Bezmialem Vakif University, School of Medicine, Pediatric Neurology, istanbul, Turkey; ${ }^{4}$ Institute of Biomedical Engineering, Bogazici University, Istanbul, Turkey; ${ }^{5}$ Istanbul University, Aziz Sancar Institute of Experimental Medicine, Neuroscience, Istanbul, Turkey; ${ }^{6}$ Istanbul UniversityCerrahpasa, Cerrahpasa Medical School, Neuroradiology, ístanbul, Turkey;

${ }^{7}$ Istanbul University-Cerrahpasa, Cerrahpasa Medical School, Pediatric Neurology, istanbul, Turkey

Background: Systemic lupus erythematosus (SLE) is a chronic inflammatory disease characterized by the presence of various autoantibodies. Unnoticed and progressive cognitive impairment may develop in the course of disease even without overt neuropsychiatric (NP) features. Some authors attributed this mild impairment to the immune-mediated myelinopathy. Evidence exists that myelin oligodendrocyte glycoprotein (MOG) might act as a mediator of interactions between myelin and the immune system.

Objectives: To detect the role of MOG-Ab in neurologic manifestations of childhood-onset SLE (CSLE) and to better delineate the actual grade of cognitive dysfunction by neurocognitive tests in patients without overt NP features (non-NPSLE). Moreover, we aimed to identify the localization of pathology at molecular level by analyzing the presence of any correlation between various metabolite peaks in MR spectroscopy (MRS) and the severity of cognitive dysfunction in non-NPSLE children.

Methods: MOG-Ab levels were studied in all healthy subjects $(n=28)$ and in all patients with (NPSLE =9) and without (non-NPSLE=36) overt neuropsychiatric manifestations. All of the non-NPSLE group and healthy group underwent brain MR and MRS examination. However, only 20 subjects in each group met the MRS imaging standards for evaluation. In the nonNPSLE group, 29 cSLE patients were further evaluated by neurocognitive tests. Sixteen children with non-NPSLE were assessed by both MRS and neurocognitive tests.

Results: The mean age of the SLE patients at study time was 16.22 3.22 years. MOG-Ab was detected positive neither in CSLE nor in healthy group.

In children with non-NPSLE, verbal IQ ranged from 40 to 108 (mean: $79.06 \pm 17.66$ ), performance IQ ranged from 42 to 111 (mean: 92.03 \pm 16.28 ), and full-scale IQ ranged from 40 to 106 (mean: 84.31 \pm 16.39 ). There were 15 patients $(51 \%)$ in non-NPSLE group with a full-scale IQ under 85. There was no significant difference between the non-NPSLE group and healthy subjects in terms of choline, $\mathrm{N}$-aspartic acid (NAA), creatine, NAA/creatine and choline/creatine.

A negative correlation was observed between the NAA/Creatine level of the left frontal white matter in MRS and the stroop test time (measures the ability to shift a perceptual set with changing demands which is a function of the frontal lobes) $(p=0.015 ; r=0.596)$.

Conclusion: More than a half of our patients in non-NPSLE group were found to have a full-scale IQ under 85. Cognitive impairment may develop insidiously in CSLE children even without any overt symptom or sign. There was no association of MOG-Ab with CSLE, whether neuropsychiatric manifestations present or not. A causal relationship between immune-mediated myelinopathy and neuropsychiatric involvement/cognitive impairment could not be suggested, since there has been no patient with positive MOG-Ab and there has been no difference in choline, cholin/creatine between CSLE patients and healthy subjects. Decrease in the NAA Creatine level of the left frontal white matter in MRS, which is a finding of neuronal loss, may be used as a first sign of cognitive impairment in patients with CSLE.

Disclosure of Interests: : None declared

DOI: 10.1136/annrheumdis-2019-eular.7658 